\title{
Tsafon
}

Revue d'études juives du Nord

$73 \mid 2017$

Connaissance de la Shoah : témoignages, enseignements, visites

\section{Les voyages d'étude à Auschwitz du Mémorial de la Shoah}

\section{Mathias Orjekh et Bertrand Bossy}

\section{(2) OpenEdition}

\section{Journals}

Édition électronique

URL : https://journals.openedition.org/tsafon/295

DOI : $10.4000 /$ tsafon.295

ISSN : 2609-6420

Éditeur

Association Jean-Marie Delmaire

Édition imprimée

Date de publication : 1 juin 2017

Pagination : 119-128

ISSN : $1149-6630$

Référence électronique

Mathias Orjekh et Bertrand Bossy, «Les voyages d'étude à Auschwitz du Mémorial de la Shoah », Tsafon [En ligne], 73 | 2017, mis en ligne le 31 mai 2018, consulté le 24 juin 2021. URL : http:// journals.openedition.org/tsafon/295; DOI : https://doi.org/10.4000/tsafon.295 


\title{
Les voyages d'étude à Auschwitz du Mémorial de la Shoah
}

Mathias Orjekh*

Bertrand Bossy **

\author{
Première partie : Le Mémorial de la Shoah, \\ ses activités pédagogiques et les voyages d'étude à Auschwitz
}

par Mathias Orjekh

La transmission de l'histoire et de la mémoire de la Shoah sont deux éléments fondamentaux et constitutifs du Mémorial de la Shoah, inauguré le 27 janvier 2005. Il s'inscrit dans la continuité des activités du Centre de Documentation Juive Contemporaine (C.D.J.C.) et du Mémorial du Martyr Juif Inconnu, créés respectivement en 1943 et en 1956, dont il est une émanation et dont il poursuit les objectifs et les actions.

Ainsi lors de sa création par Isaac Schneersohn, en avril 1943, à Grenoble dans la zone d'occupation italienne, le C.D.J.C. a pour vocation de rassembler des documents sur les persécutions dont les Juifs sont victimes. Ces archives seront d'ailleurs présentées, comme autant de preuves, lors du procès de Nuremberg.

Adossé au C.D.J.C., dont les bureaux sont installés rue Geoffroy l'Asnier à Paris, le Mémorial du Martyr Juif Inconnu est créé en 1956. Premier mémorial en hommage aux Juifs assassinés durant la Shoah, sa

\footnotetext{
${ }^{*}$ Coordinateur des voyages d'étude au Mémorial de la Shoah.

** Professeur d'histoire-géographie au lycée Fernand Renaudeau-Cholet (49).
} 
vocation est plus mémorielle; même si une petite exposition trouve également place dans le bâtiment.

De fait, depuis 2005, le Mémorial de la Shoah est résolument tourné vers la transmission, notamment vers les élèves, et la formation des enseignants. Des activités pédagogiques se développent continuellement, parallèlement à sa mission originelle: porter la mémoire des victimes.

\section{Les activités pédagogiques proposées par le Mémorial de la Shoah}

Ainsi, le Mémorial de la Shoah a accueilli 224970 visiteurs en 2015 dont 38000 jeunes, venus à 94\% dans le cadre scolaire. De nombreuses activités pédagogiques sont proposées aux enseignants.

Outre la visite de l'exposition permanente du Mémorial, il est possible de faire des visites thématiques sur des sujets comme «les différentes formes de résistance », « l'antisémitisme nazi » ou « la France de Vichy» par exemple. Des parcours de mémoire sont également organisés. Les élèves complètent ainsi leur visite du Mémorial par la découverte d'un lieu de mémoire de la Shoah ou des traces de la présence des Juifs à Paris : les mémoriaux de la déportation et du Vel d'Hiv, les camps annexes de Drancy, le Marais juif et les Judéo-espagnols dans le $\mathrm{XI}^{\mathrm{e}}$ arrondissement, etc.

Plusieurs ateliers ont également été développés à destination du public scolaire. Ceux-ci permettent de mettre l'accent sur des sujets particuliers : les enfants cachés, la résistance juive, les caricatures, les préjugés...

Les attentats de janvier 2015 et l'impossibilité ponctuelle pour les classes de se rendre à Paris ont entraîné une réorientation de la proposition pédagogique du Mémorial. Plusieurs activités, en particulier les ateliers, sont ainsi développées hors des murs de l'institution et sont maintenant également mis en place au sein même des établissements scolaires.

Depuis plus de dix ans, le Mémorial de la Shoah organise également des voyages d'étude à Auschwitz. Ceux-ci sont articulés autour d'un projet pédagogique d'envergure qui mobilise une classe ou un groupe d'élèves volontaires sur la quasi-totalité de l'année scolaire et s'adressent aux élèves des lycées généraux, technologiques, professionnels et agricoles, et aux apprentis. 
Ces voyages d'étude à Auschwitz ont concerné, en 2016, environ 2000 élèves d'Ile de France et de six autres régions, avec lesquelles le Mémorial a noué un partenariat: Alsace, Midi-Pyrénées, Nord/Pas-deCalais, Normandie, Pays de la Loire et Provence-Alpes-Côte d'Azur. Ce dispositif a été initié au niveau national en 2004 par la Fondation pour la Mémoire de la Shoah.

Les enseignants souhaitant participer à ce programme proposent, à l'appel du Mémorial de la Shoah, des Régions et des Rectorats partenaires, un projet pédagogique autour de l'histoire ou de la mémoire de la Shoah; sur la base duquel les classes sont retenues par un jury composé des différentes institutions partenaires.

Ces projets peuvent être de nature très diverses mais les enseignants sont invités à travailler notamment sur l'histoire locale, afin de permettre à leurs élèves de mieux appréhender le fait que les persécutions ont pu toucher des Juifs proches de leur univers géographique.

Ce travail d'histoire est indispensable car le voyage d'étude à Auschwitz n'est pas une finalité en soit mais doit, au contraire, être une étape dans la réalisation du projet pédagogique. L'objectif de ces voyages d'étude est de permettre aux élèves de se rendre sur le lieu où a été déportée et assassinée une grande partie des Juifs de France, afin de mieux comprendre le processus d'assassinat mis en place par les nazis mais aussi les enjeux historique et mémoriel du camp d'Auschwitz.

La visite leur permet également de suivre l'itinéraire des personnes dont le parcours sert souvent de base aux projets pédagogiques. C'est le cas par exemple des élèves du lycée Saint-Louis de Saumur (49) qui au cours de l'année 2014-2015 avaient travaillé sur le parcours de la famille Abraham-Meier réfugiée à Chênehutte-Les-Tuffeaux, près de leur ville, et déportés en $1942^{1}$ ou des élèves du lycée Anguier de Eu (76) qui ont étudié la vie de la famille Salmona dont les membres résidaient au Tréport avant leur arrestation et leur déportation en 1942 et $1943^{2}$.

Le Mémorial apporte un soutien technique, pédagogique et historique durant toute la phase de préparation au voyage. La préparation

\footnotetext{
${ }^{1}$ Ce projet a donné lieu à la production, par les élèves, d'une exposition sur la famille Abraham-Meier. Grâce notamment à l'implication du maire délégué de ChênehutteTrèves-Cunault, une stèle à la mémoire des membres de la famille a également été inaugurée en octobre 2016 à côté du monument aux morts du village, en présence des élèves.

${ }^{2}$ À l'issue de leurs recherches sur la famille Salmona, les élèves ont réalisé un film suivant le procédé dit du draw my life présentant leur parcours.
} 
des classes retenues est en effet un des piliers de la qualité du travail des élèves et de leur engagement.

Ainsi des livrets pédagogiques destinés aux élèves et aux professeurs sont distribués à chacun des participants. Ils apportent un éclairage historique sur les différentes étapes de la Shoah, le complexe d'Auschwitz et la « Solution finale » en France.

En outre, la majorité des classes se rend au Mémorial de la Shoah pour une visite axée spécifiquement sur la préparation au voyage. Celleci est complétée par la rencontre avec un témoin ancien déporté d'Auschwitz, lorsque cela est encore possible.

Si la venue des classes ne s'avère pas réalisable, les coordinateurs des voyages d'étude se déplacent dans les établissements pour animer des séances de travail et de réflexion, autour de l'histoire du nazisme et de la Shoah notamment. Des ateliers et des prêts d'exposition sont également proposés.

\section{Le déroulement du voyage d'étude à Auschwitz}

À l'exception d'établissements de certaines régions, le voyage d'étude se déroule sur une journée. Durant cette journée, les classes sont encadrées sur les sites de Birkenau et d'Auschwitz par un guide francophone du Musée d'Auschwitz et un accompagnateur-historien du Mémorial de la Shoah.

La visite repose ainsi sur une problématisation tout au long du parcours au camp d'Auschwitz-Birkenau mettant en relief, d'une part la logique de mise à mort du plus grand nombre des déportés juifs à l'arrivée des convois avec leur transfert immédiat vers les chambres à gaz, et d'autre part l'entrée dans le système concentrationnaire pour la minorité des déportés « sélectionnés » pour le travail.

Le parcours peut également évoluer en fonction de la présence ou non d'un rescapé et de son histoire personnelle, afin de privilégier les lieux qu'il a connus au camp, mais aussi en fonction des souhaits exprimés par les enseignants selon la teneur de leur projet pédagogique.

Ainsi après une prise de parole, durant le transfert depuis l'aéroport de Cracovie, au cours de laquelle des indications à caractère historique sur la situation du site d'Auschwitz, la Shoah et les Juifs de France durant la Seconde Guerre mondiale sont données, la visite débute par la découverte du camp de d'Auschwitz-II Birkenau. Le choix de commencer par ce site et non par celui d'Auschwitz-I se justifie, même si 
cet ordre ne respecte pas la chronologie de la construction des camps, par le fait qu'il permet de mettre en relief le processus d'assassinat des Juifs qui s'est déroulé majoritairement et massivement sur ce lieu.

Selon le temps effectif, les espaces visités sont la "Judenrampe », la «Bahnrampe », les Krematorium II à V, les Bunker I et II, le «Kanada», la «Zentralsauna », le camp de quarantaine ou le camp des femmes. Cette première partie de visite s'achève par un moment de recueillement devant le monument international, au cours duquel les rescapés, les représentants des institutions partenaires et ceux des classes sont invités à prendre la parole.

Après une courte pause, la visite reprend sur le site d'Auschwitz-I, comprenant notamment les expositions présentes dans le «Pavillon français » (Block 20) et les Blocks 4 («Extermination») et 5 («Les preuves matérielles du crime »), ainsi que le mur des fusillés et le Krematorium I.

Le trajet de retour à l'aéroport permet un moment d'échanges et de réflexion entre les participants, les accompagnateurs et les rescapés.

Plus ponctuellement, il arrive que le Mémorial de la Shoah organise également des voyages d'étude d'une durée de plusieurs jours. La visite du complexe d'Auschwitz-Birkenau est alors complétée par la découverte de l'ancien quartier juif et du site du ghetto de Cracovie, ville située non loin du camp.

Ainsi les élèves réalisent un parcours dans la ville de Cracovie afin de découvrir l'importance historique et culturelle du judaïsme en Pologne, tout particulièrement dans cette ville, où environ 60000 Juifs (soit près de $25 \%$ de la population totale) vivaient avant la Seconde Guerre mondiale. La visite de l'ancien quartier juif (Kazimierz) et de plusieurs synagogues permet de découvrir à la fois les pratiques religieuses et la culture juive dans sa diversité. Les élèves peuvent appréhender la place centrale de Cracovie dans le judaïsme d'Europe de l'Est, notamment au $\mathrm{XVI}^{\mathrm{e}}$ siècle.

Un parcours dans le quartier de Podgorze permet également aux élèves de se confronter aux vestiges du ghetto où les nazis ont enfermé 15000 à 20000 Juifs, avant leur déportation vers les centres de mise à mort de Belzec et puis d'Auschwitz-Birkenau.

La visite est souvent complétée par la découverte du Musée historique de Cracovie sous l'Occupation, installé dans l'ancienne usine d'Oskar Schindler. 


\section{La finalisation et la restitution des projets pédagogiques}

Après le voyage d'étude à Auschwitz, les classes participantes engagent la réalisation des travaux de restitution prévus dans leurs projets pédagogiques. Durant cette période de finalisation des projets, le Mémorial de la Shoah poursuit l'accompagnement pédagogique et historique des enseignants et de leurs élèves. Il apporte notamment son aide en fournissant des documents d'archives et des photographies qui permettent d'illustrer les travaux de restitution.

Les productions des élèves peuvent être réparties pour l'essentiel en quatre grandes catégories : expositions, créations artistiques, supports multimédia (films, sites internet, diaporamas, émissions de radio) et productions écrites (journaux, carnets de bord, plaquettes historiques et bandes dessinées). Cette phase de finalisation permet de faire la synthèse des connaissances acquises et débouche sur l'organisation de diverses manifestations: inaugurations d'expositions, projections-rencontres, interventions devant d'autres classes, journées portes ouvertes, présentations des travaux aux parents d'élèves...

\section{La réaction des enseignants}

À la fin de l'année scolaire, une réunion de bilan rassemble les enseignants coordinateurs venant de toutes les académies concernées à l'échelle nationale. Afin de préparer cette réunion, ils sont invités à donner leur avis par l'intermédiaire de questionnaires d'évaluation.

Dans l'ensemble, les professeurs se déclarent satisfaits de l'aide apportée par le Mémorial de la Shoah dans la préparation du voyage d'étude et la mise en œuvre de leur projet pédagogique. Ils soulignent la disponibilité de leurs interlocuteurs et la pertinence des réponses obtenues.

De même, le déroulement et le contenu de la journée préparatoire sont favorablement jugés par une large majorité des enseignants. La découverte du Mémorial de la Shoah ou les interventions et ateliers proposés apparaissent comme des étapes essentielles de la préparation du voyage d'étude.

Les enseignants soulignent l'importance des projets pédagogiques qui permettent «de rendre tangibles les éléments étudiés en classe » (V P., professeur d'histoire-géographie à Nîmes [30]). Ils considèrent que 
la réalisation de ces projets et le voyage d'étude à Auschwitz sont des expériences qui entraînent « une plus grande maturité des élèves » (S K., professeur de lettres-histoire à Cernay [68]) et permettent également de « souder les classes grâce à un projet fédérateur qui occupe l'essentiel de l'année » (E M., professeur d'histoire-géographie à Strasbourg [67]).

Le voyage d'étude satisfait la totalité des enseignants qui soulignent souvent la présence, la disponibilité et la pertinence des interventions des historiens accompagnateurs du Mémorial de la Shoah.

Comme un résumé des objectifs du Mémorial, L J-L, professeur d'histoire-géographie à Bouxwiller [67], indique enfin que :

...les jeunes ont souvent du mal à être dans la réalité du moment dans lequel ils vivent et il est encore plus difficile souvent de leur transmettre un passé qui apparaît bien lointain et abstrait (...) [Le Mémorial de la Shoah leur a] permis ainsi de mieux comprendre le passé, de l'intégrer dans leur culture. Le déplacement à Auschwitz a fait de ces jeunes lycéens des hérauts qui ne pourront que poursuivre cette œuvre pour le vivre ensemble, pour la paix.

$* * * * * * * * * * * * * * * *$ 


\title{
Seconde partie : Un voyage d'étude à Auschwitz, expérience croisée enseignant-élèves
}

\author{
par Bertrand Bossy ${ }^{3}$
}

Professeur d'histoire-géographie au lycée Fernand Renaudeau de Cholet (Académie de Nantes), j'ai mené trois projets pédagogiques qui ont permis à cinq classes de Première ou de Terminale d'effectuer un voyage d'étude à Auschwitz grâce au partenariat entre le Mémorial de la Shoah, la Région Pays de la Loire et le Rectorat de Nantes.

- «Être un enfant juif à Cholet (1940-1944)»: Dossier pédagogique et Exposition.

Projet pluriannuel commencé en 2005-2006 avec une Terminale Littéraire (projet pluridisciplinaire histoire-géographie et philosophie) et finalisé en 2010-2011 avec une Première Littéraire (projet pluridisciplinaire histoire-géographie, français et atelier théâtre).

- «Un aller sans retour: sur les traces d'Anne Loew (19381944)» : Carnet d'enquête (24 pages).

Projet réalisé en 2014-2015 avec une Première Économique et Sociale (projet pluridisciplinaire histoire-géographie, français et arts appliqués).

- «Mémoire dans la ville : Cholet et la Shoah»: Livre Blanc (rédaction et mise en œuvre).

Projet pluriannuel commencé en 2015-2016 avec une Terminale Économique et Sociale (projet pluridisciplinaire histoire-géographie, CDI et EPS. Rédaction du Livre Blanc) et poursuivi en 2016-2017 avec une Première Économique et Sociale (projet pluridisciplinaire histoiregéographie, CDI et EPS. Mise en œuvre des propositions du Livre Blanc).

\footnotetext{
${ }^{3}$ Bertrand Bossy est l'auteur de Histoire de la déportation des Juifs dans les Mauges, Le Crèche (79), Geste éditions (collection Pays d'Histoire), 2014, 264 p.
} 
Évoquer cette expérience de cinq voyages d'étude à Auschwitz avec plus d'une centaine d'élèves c'est inévitablement se poser la question suivante : pourquoi emmener des élèves à Auschwitz ?

Les élèves du lycée (recrutement milieu urbain et rural, classes moyennes et familles modestes), pour leur immense majorité, manifestent une adhésion très forte aux projets dans leur préparation en amont. La journée d'étude à Auschwitz est très éprouvante pour eux, c'est ce que révèle à chaque fois la séance - indispensable - de débriefing organisée à leur retour au lycée. C'est davantage Auschwitz-I qui suscite l'émotion chez les élèves (choc de la confrontation aux objets de la vie quotidienne du Musée d'Auschwitz). C'est pourquoi la « visite» d'Auschwitz-Birkenau est absolument nécessaire selon eux pour comprendre la spécificité et la complexité d'Auschwitz. Aller là où « il n'y a rien à voir»-Bunker II, zone des bûchers, zone des étangsmares - s'impose impérativement selon eux pour la compréhension du « projet» des nazis.

Le voyage d'étude à Auschwitz provoque également, au-delà des seuls élèves, une implication plus large en suscitant des discussions, des échanges intergénérationnels au sein des familles. Ainsi le cas de cet élève qui a appris tout récemment l'existence d'un Juste parmi les Nations dans sa famille ${ }^{4}$. L'adhésion des familles se traduit aussi par leur participation, y compris en période de vacances scolaires, à la cérémonie commémorative de dernier dimanche d'avril, seule cérémonie à caractère officiel à Cholet.

En tant qu'enseignant, accompagner des élèves sur les sites d'Auschwitz-I et II permet une approche pédagogique non plus seulement fondée sur des documents-sources, pratique commune en classe, mais également sur le ressenti des élèves, paramètre supplémentaire et indissociable, selon moi, de l'enseignement de la Shoah.

Enfin, pour les élèves comme pour les professeurs, ces voyages d'étude ont nourri une réflexion sur leur responsabilité dans la transmission de cette mémoire à l'échelle locale.

Je terminerai par évoquer le cas de l'une de mes anciennes élèves, Glynis Boré, et devenue depuis ma collègue professeure-documentaliste.

\footnotetext{
${ }^{4}$ Les voyages d'étude permettent également de libérer la parole entre enseignants : exemple d'une collègue professeure-documentaliste évoquant en 2016 - soit 10 ans après le premier projet - son grand-père juif déporté pour fait de résistance et rescapé des camps de concentration.
} 
Volontaire dans le projet pédagogique de cette année scolaire, elle accompagnera avec moi, en mars prochain, une classe à Auschwitz qu'elle avait donc découvert en 2011. Les échanges avec ma jeune collègue dans la préparation de ce nouveau projet m'ont confirmé les impacts, même s'ils sont difficiles à appréhender, de ces voyages d'étude à Auschwitz.

Découvrir, réfléchir et transmettre, voilà des objectifs atteints avec ces différents projets. 